\title{
Optimal Rate Allocation in Overlay Content Distribution
}

\author{
Chuan Wu and Baochun Li \\ Department of Electrical and Computer Engineering \\ University of Toronto \\ $\{$ chuanwu, bli\}@eecg. toronto.edu
}

\begin{abstract}
This paper addresses the optimal rate allocation problem in overlay content distribution for efficient utilization of limited bandwidths. We systematically present a series of optimal rate allocation strategies by dividing our discussions into four typical scenarios. Based on application-specific requirements, these scenarios reflect the contrast between elastic and streaming content distribution, with either per-link or per-node capacity constraints. In each scenario, we show that the optimal rate allocation problem can be formulated as a linear optimization problem, which can be solved efficiently in a fully distributed fashion. In simulations, we investigate the convergence of our distributed algorithms in both static and dynamic networks, and demonstrate their efficiency.
\end{abstract}

Keywords: Overlay Network, Rate Allocation, Optimization.

\section{Introduction}

In recent years, content distribution with overlay networks has been proposed to offer more efficient bandwidth usage than that with multiple unicast sessions. To achieve better bandwidth utilization and failure resilience, overlay content distribution over mesh topologies has become typical in most recent proposals, which features parallel downloading from multiple overlay nodes.

However, due to the limitation of bandwidth capacities in overlay networks, a critical question remains to be answered in any such content distribution scheme: What is the best way to select upstream peers and allocate flow rates in an overlay topology, such that content can be most efficiently distributed?

To effectively address this question, it is necessary to consider that different content distribution applications may have different optimality goals and constraints for their rate allocation strategies. The content to be distributed can be classified into two broad categories: elastic content (e.g., bulk data files), and streaming content with specific bit rate requirements (e.g., media streaming). In the case of distributing elastic content, such as file downloading, it is important to optimally select upstream nodes and allocate flow rates so that throughput of content distribution can be maximized. In the case of distributing media streams, the required streaming rate needs to be sustained for all receivers in active sessions. Besides, in both cases, capacity constraints in overlay networks may lie in 
the overlay links (link capacity constraints), due to limited available bandwidth along the link, or the overlay nodes (node download/upload capacity constraints), caused by limited node download/upload capacities.

In this paper, we consider both types of content and both assumptions of capacity constraints, and systematically present a series of optimal rate allocation strategies in four content distribution scenarios. We show that in each scenario, the optimal rate allocation problem can be modeled into a linear optimization problem, for which efficient and fully decentralized solutions exist.

The remainder of the paper is as follows. In Sec. 2 and Sec. 3. we motivate the optimization formulations in elastic and streaming content distribution scenarios, respectively, and present efficient distributed solution algorithms. We discuss practical concerns of applying the algorithms in Sec. 4. Simulation results are presented in Sec. 5. We discuss related work and conclude the paper in Sec. 6] and Sec. [7, respectively.

\section{2 eBurst: Distribution of Elastic Content}

In this paper, we consider content distribution sessions in mesh overlay topologies, consisting of one data source $S$ and a set of receivers in $T$. Each receiver is served by one or more upstream nodes, and may serve one or more downstream nodes. Such a topology can be modeled as a directed graph $G=(N, A)$, where $N$ is the set of overlay nodes and $A$ is the set of overlay links. We have $N=S \cup T$.

To distribute elastic content, it is always desirable to achieve maximum throughput in order to minimize the total time to completion. The problem is: How do we optimally allocate rates on each overlay link to maximize throughput? We show that such a problem, referred to as eBurst, can be formulated as linear programs.

In order to better characterize the multicast flow of a content distribution session, we resort to the notion of conceptual unicast flows [1] in formulating the linear programs. A multicast content distribution flow can be conceptually viewed as consisting of multiple unicast flows from the source to each of the receivers. These conceptual unicast flows co-exist in the overlay without contending for capacities, and the actual delivery rate on an overlay link is the maximum of the rates of all the conceptual flows going through the link. In formulating the linear programs, the utilization of conceptual unicast flows is useful to capture the inherent property of a multicast flow, as the conceptual unicast flows follow the nice property of flow conservation at each intermediate node, while the multicast flows do not.

\section{1 eBurst with Link Capacity Constraints}

We first consider the assumption that capacity constraints lie in the overlay links, which is the case when the bottleneck is in the core of the overlay, such as transcontinental links. Let $u_{i j}$ be the capacity of overlay link $(i, j)$. $R$ denotes the throughput of the content distribution session, i.e., the aggregate receiving rate at each participating peer. $x_{i j}$ is the delivery rate on link $(i, j)$. Let $f^{t}$ denote the conceptual unicast flow from source $S$ to a receiver $t,\left|f^{t}\right|$ be its flow rate, 
Table 1. eBurst LCC LP

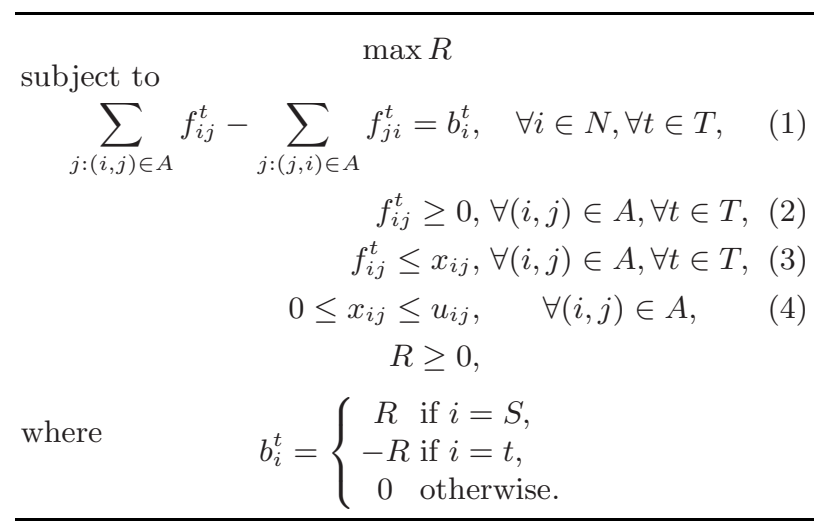

and $f_{i j}^{t}$ be the rate of $f^{t}$ flowing through link $(i, j)$. The eBurst problem with Link Capacity Constraints (LCC) can be formulated as the linear program in Table 1, referred to as the eBurst $L C C$ LP.

In this LP, (1) and (2) model each conceptual flow $f^{t}$ as a valid network flow, following flow conservations. (3) represents the relation between conceptual flow rates and the actual delivery rate on each link, which is further constrained by link capacities in (4).

There exists an efficient combinatorial algorithm to solve the eBurst LCC LP. By reformulating constraints (2), (3) and (4) as

$$
0 \leq f_{i j}^{t} \leq u_{i j}, f_{i j}^{t} \leq x_{i j} \leq u_{i j}, \forall(i, j) \in A, \forall t \in T
$$

we notice that this LP can be decomposed into $|T|$ maximum flow problems, each corresponding to one conceptual unicast flow $f^{t}, \forall t \in T$. Therefore, this LP can be solved by computing maximum flows from the source to each of the receivers, and then delivery rate $x_{i j}$ is decided as the maximum of the rates of all the maximum conceptual flows on $(i, j)$. Since the maximum flow problem can be solved by distributed algorithms, such as push-relabel algorithm [2], we are able to derive an efficient decentralized algorithm for the LP, as given in Table2.

Table 2. Distributed algorithm for eBurst LCC LP

$\overline{1 .}$ Compute maximum flow $f^{t}$ from $S$ to $t, \forall t \in T$, with distributed push-relabel algorithm.

2. Compute the maximum throughput $R=\min _{t \in T}\left|f^{t}\right|$.

3. Compute optimal rates $x_{i j}=\max _{t \in T} f_{i j}^{t}, \forall(i, j) \in A$.

\section{2 eBurst with Node Capacity Constraints}

When bandwidth bottlenecks occur at the "last-mile" links to the overlay nodes, it is more appropriate to model capacity constraints at each node rather than 
Table 3. eBurst $N C C$ LP

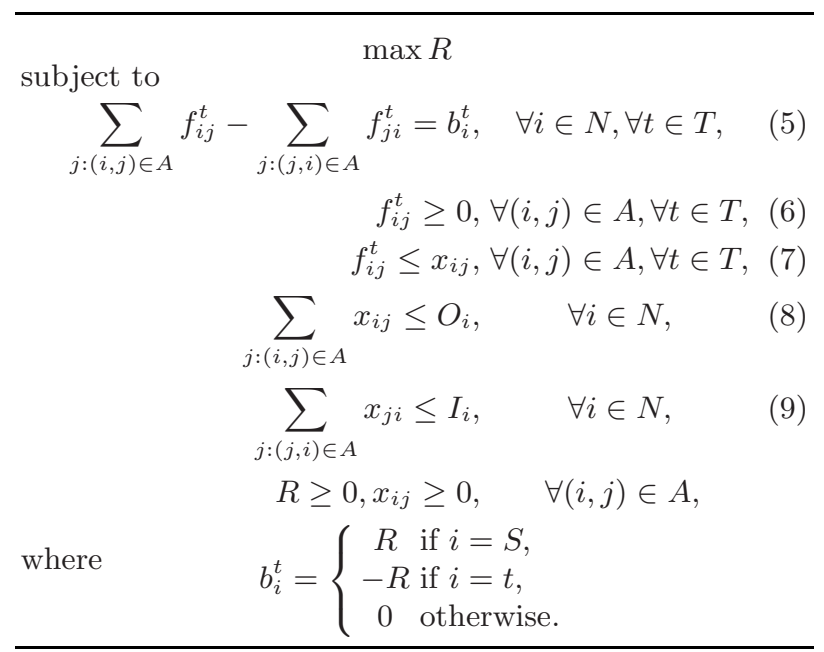

each link, with maximum upload and download capacities. For node $i$, let $O_{i}$ be its upload capacity and $I_{i}$ be its download capacity. The linear program with Node Capacity Constraints (NCC) is formulated in Table 3 referred to as the eBurst NCC LP.

This LP regulates delivery rates on the overlay links using node capacities in (8) (9), rather than link capacities in (44). It is not readily decomposable to known combinatorial optimization problems. To obtain a distributed algorithm, we apply Lagrangian relaxation and design the corresponding subgradient algorithm, which is an efficient LP solution technique and can be naturally implemented in a distributed manner.

We have derived a fully decentralized algorithm by applying Lagrangian relaxation to the dual of the eBurst NCC LP. Due to space limit, we only provide the main idea to develop the algorithm. For complete details, interested readers are referred to our technical report [3].

We first note that, if we can decide the set of optimal delivery rates, $x_{i j}$, $\forall(i, j) \in A$, that satisfy (8) (9), the eBurst NCC LP boils down to an eBurst $L C C$ problem. In order to obtain the optimal values for primal variables $x_{i j}$, we investigate the variable-constraint correspondence between an LP and its dual, i.e., each primal variable corresponds to one dual constraint. When Lagrangian relaxation is applied to the dual LP, a primal variable is actually the same as the Lagrangian multiplier associated with its corresponding dual constraint. We further understand that with the Lagrangian relaxation technique, the optimal values for the Lagrangian multipliers can be obtained by the subgradient algorithm. Therefore, to acquire $x_{i j}$, we solve the dual LP of the eBurst NCC LP in Table 3 with Lagrangian relaxation and subgradient algorithm, by relaxing the set of dual constraints corresponding to the primal variables $x_{i j}, \forall(i, j) \in A$. 
Table 4. Distributed algorithm for eBurst NCC LP

1. Initialize rates $x_{i j}[0], \forall(i, j) \in A$, to non-negative values.

2. Repeat the following iteration until the sequence $\{x[k]\}$ converges to $x^{*}$ :

(1) With $x_{i j}[k]$ as the upper bound of the delivery rate on link $(i, j), \forall(i, j) \in A$, compute the maximum flow from $S$ to $t, \forall t \in T$, with the distributed push-relabel algorithm;

(2) Update $x$ by

— Compute $x^{\prime}=x[k]+\theta[k] \sum_{t \in T} z^{t}[k]$, where $\theta[k]=a /(b+c k), a>0, b \geq$ $0, c>0$, and for all $(i, j) \in A$,

$$
z_{i j}^{t}[k]=\left\{\begin{array}{c}
1 \text { if edge }(i, j) \text { is in the min cut of the } \\
\text { minimum of all maximum flows from } \\
S \text { to } t, \forall t \in T \\
0 \text { otherwise. }
\end{array}\right.
$$

- Project $x^{\prime}$ onto the feasible simplex

$$
P^{\prime}=\left\{x \mid \sum_{j:(i, j) \in A} x_{i j} \leq O_{i}, \sum_{j:(j, i) \in A} x_{j i} \leq I_{i}, \forall i \in N, x_{i j} \geq 0, \forall(i, j) \in A\right\}
$$

by $x_{i j}[k+1]=\min \left(x_{i j}^{\prime}, \frac{x_{i j}^{\prime}}{\sum_{m:(i, m) \in A} x_{i m}^{\prime}} O_{i}, \frac{x_{i j}^{\prime}}{\sum_{m:(m, j) \in A} x_{m j}^{\prime}} I_{j}\right), \forall(i, j) \in A$.

$\rightarrow$ Optimal delivery rates obtained.

3. With $x_{i j}^{*}$ as the link capacity on link $(i, j), \forall(i, j) \in A$, compute the maximum flow $f^{t}$ from $S$ to $t, \forall t \in T$, with the distributed push-relabel algorithm.

4. Compute the maximum throughput $R=\min _{t \in T}\left|f^{t}\right|$.

$\rightarrow$ Maximum content distribution throughput obtained.

We also observe that the LP in Table 3 has the underlying structure of maximum flow problems. Therefore, due to the primal-dual relationship between max-flow and min-cut linear programs, its dual LP has the underlying structure of min-cut problems, which we can utilize when solving the dual LP with subgradient algorithm. The complete distributed algorithm is shown in Table 4 .

This distributed algorithm has nice combinatorial interpretations. Starting from some initial feasible delivery rates, the optimal rates are derived iteratively. In each iteration, we increase rates on links in the current minimum cut of the network, i.e., links that are saturated with currently allocated rates, and always guarantee node capacity constraints are satisfied by projecting increased rates onto the feasible simplex $P^{\prime}$. After this projection, the bandwidth share for non-saturated links, i.e., links that are not in the minimum cut, is reduced while that for saturated links is increased. This refinement repeats itself until the optimal rate allocation on all the links is achieved.

\section{3 sBurst: Distribution of Streaming Content}

Real-time content streaming, such as live multimedia or stocks quotes, usually demands a fixed streaming rate, $r$, to sustain the streaming session. For 
these applications, instead of maximizing throughput, it is desirable to optimize rate allocations to minimize the total cost of streaming, while guaranteeing the streaming rate $r$. More rigorously, if we use $c_{i j}$ to denote the streaming cost on an overlay link $(i, j)$, our objective is to minimize $\sum_{(i, j) \in A} c_{i j} x_{i j}$. When $c_{i j}$ represents per-link delay, the optimal rate allocation minimizes total delay of the session. When all $c_{i j}$ 's are 1 , the total bandwidth usage is minimized and thus the best bandwidth efficiency is achieved by the optimization. Henceforth, this optimization problem is referred to as sBurst.

\section{1 sBurst with Link Capacity Constraints}

The sBurst problem with link capacity constraints, referred to as sBurst $L C C$ $\mathrm{LP}$, is formulated in Table 5 .

Table 5. sBurst LCC LP

\[ \begin{array}{l}\min \sum_{(i, j) \in A} c_{i j} x_{i j} \\ \sum_{j:(i, j) \in A} f_{i j}^{t}-\sum_{j:(j, i) \in A} f_{j i}^{t}=b_{i}^{t}, \quad \forall i \in N, \forall t \in T, \quad(10) \\ f_{i j}^{t} \geq 0, \forall(i, j) \in A, \forall t \in T,(11) \\ f_{i j}^{t} \leq x_{i j}, \forall(i, j) \in A, \forall t \in T,(12)\end{array} \]
$0 \leq x_{i j} \leq u_{i j}, \quad \forall(i, j) \in A, \quad$ (13)
where $\begin{gathered}r \text { if } i=S, \\ -r \text { if } i=t, \\ 0 \text { otherwise. }\end{gathered}$
$b_{i}^{t}=$

This LP can be solved with Lagrangian relaxation and subgradient algorithm, by relaxing constraint group (12). Associating Lagrangian multipliers $\mu_{i j}$ with (12), we obtain the Lagrangian dual of the sBurst LCC LP:

$$
\max _{\mu \geq 0} L(\mu)
$$

where

$$
L(\mu)=\min _{P} \sum_{(i, j) \in A} x_{i j}\left(c_{i j}-\sum_{t \in T} \mu_{i j}^{t}\right)+\sum_{t \in T} \sum_{(i, j) \in A} \mu_{i j}^{t} f_{i j}^{t},
$$

and polytope $P$ is defined by constraints (10) (11) (13).

The Lagrangian subproblem in (15) can be decomposed into $|T|$ shortest path problems:

$$
\min \sum_{(i, j) \in A} \mu_{i j}^{t} f_{i j}^{t}
$$


subject to

$$
\begin{array}{cc}
\sum_{j:(i, j) \in A} f_{i j}^{t}-\sum_{j:(j, i) \in A} f_{j i}^{t}=b_{i}^{t}, & \forall i \in N, \\
f_{i j}^{t} \geq 0, & \forall(i, j) \in A,
\end{array}
$$

for each $t \in T$, and a minimization problem

$$
\min \sum_{(i, j) \in A} x_{i j}\left(c_{i j}-\sum_{t \in T} \mu_{i j}^{t}\right)
$$

subject to

$$
0 \leq x_{i j} \leq u_{i j}, \forall(i, j) \in A .
$$

The shortest path problems in (16) can be efficiently solved in a distributed manner by the distributed Bellman-Ford algorithm [4]. The optimal solution to the minimization problem in (17) can be computed as follows:

$$
x_{i j}=\left\{\begin{aligned}
0 & \text { if } \sum_{t \in T} \mu_{i j}^{t} \leq c_{i j}, \\
u_{i j} & \text { if } \sum_{t \in T} \mu_{i j}^{t}>c_{i j} .
\end{aligned}\right.
$$

In each iteration of the subgradient algorithm, we solve the subproblems in (16) and (17) with the current Lagrangian multiplier values $\mu[k]$. Then we update the Lagrangian multipliers by

$$
\mu_{i j}^{t}[k+1]=\max \left(0, \mu_{i j}^{t}[k]+\theta[k]\left(f_{i j}^{t}[k]-x_{i j}[k]\right)\right), \forall(i, j) \in A, \forall t \in T,
$$

where $\theta$ is a prescribed sequence of step sizes satisfying:

$$
\theta[k]>0, \lim _{k \rightarrow \infty} \theta[k]=0 \text {, and } \sum_{k=1}^{\infty} \theta[k]=\infty .
$$

Since the primal values in the optimal solution of the Lagrangian dual are not necessarily optimal to the primal LP, we further apply the algorithm introduced by Sherali et al. [5] to recover the optimal primal values. At the $k^{\text {th }}$ iteration, we compose a primal iterate $\widetilde{f_{i j}^{t}}[k]$ via

$$
\widetilde{f_{i j}^{t}}[k]=\sum_{h=1}^{k} \lambda_{h}^{k} f_{i j}^{t}[h],
$$

where $\sum_{h=1}^{k} \lambda_{h}^{k}=1$ and $\lambda_{h}^{k} \geq 0$, for $h=1, \ldots, k$.

In our algorithm, we choose the step length sequence $\theta[k]=a /(b+c k), \forall k, a>$ $0, b \geq 0, c>0$, and convex combination weights $\lambda_{h}^{k}=1 / k, \forall h=1, \ldots, k, \forall k$. These guarantee the convergence of our subgradient algorithm; they also guarantee that any accumulation point $\tilde{f}^{*}$ of the sequence $\{\widetilde{f}[k]\}$ generated via (19) is an optimal solution to the primal problem in Table [5] [5].

Now we can design our distributed algorithm to solve sBurst $L C C$ LP. We delegate the computation tasks on link $(i, j)$ to be carried out by incident node $j$. The algorithm to be executed by each node is given in Table 6 . 
Table 6. Distributed algorithm on node $j$ for sBurst LCC LP

$\overline{\text { 1. Initialize Lagrangian multipliers } \mu_{i j}^{t}[0], \forall i:(i, j) \in A, \forall t \in T \text {, to non-negative }}$ values.

2. Repeat the following iteration until sequence $\{\mu[k]\}$ converges to $\mu^{*},\{\tilde{f}[k]\}$ converges to $\widetilde{f}^{*}: \forall i:(i, j) \in A, \forall t \in T$

(1) Compute $f_{i j}^{t}[k]$ by distributed Bellman-Ford algorithm;

(2) Compute $x_{i j}[k]$ by Eqn. (18);

(3) Compute $\widetilde{f_{i j}^{t}}[k]=\sum_{h=1}^{k} \frac{1}{k} f_{i j}^{t}[h]=\frac{k-1}{k} \widetilde{f_{i j}^{t}}[k-1]+\frac{1}{k} f_{i j}^{t}[k]$;

(4) Update Lagrangian multiplier $\mu_{i j}^{t}[k+1]=\max \left(0, \mu_{i j}^{t}[k]+\theta[k]\left(f_{i j}^{t}[k]-\right.\right.$ $\left.\left.x_{i j}[k]\right)\right)$;

3. Compute optimal rate $x_{i j}^{*}=\max _{t \in T} \widetilde{f_{i j}^{t}}{ }^{*}, \forall i:(i, j) \in A$.

Table 7. sBurst NCC LP

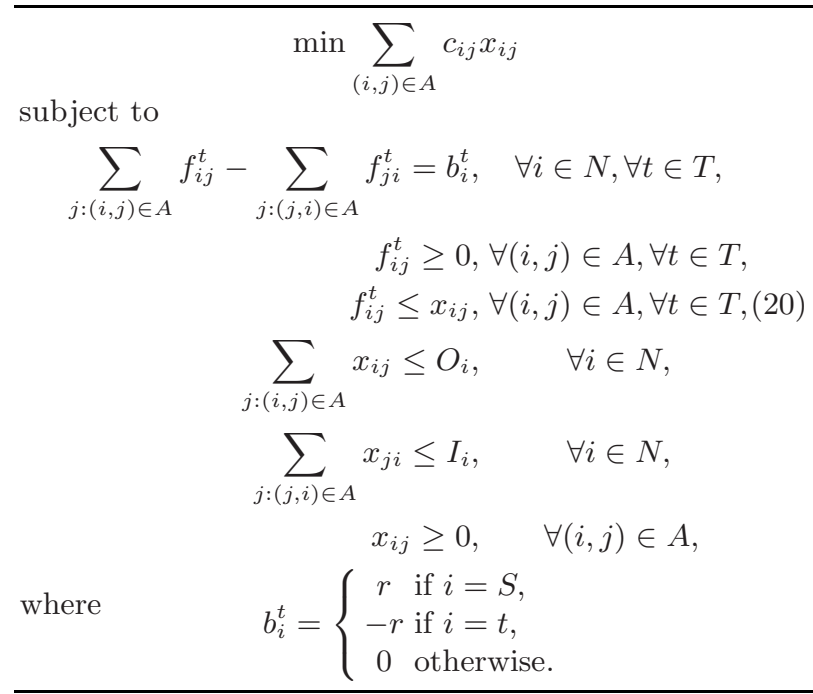

\section{2 sBurst with Node Capacity Constraints}

The sBurst problem with node capacity constraints, referred to as sBurst $N C C$ LP, is formulated in Table 7.

This LP can be solved with similar Lagrangian relaxation techniques as solving the sBurst $L C C$ LP, by relaxing (20). The only difference is that the resulting minimization subproblem is defined differently:

$$
\min \sum_{(i, j) \in A} x_{i j}\left(c_{i j}-\sum_{t \in T} \mu_{i j}^{t}\right)
$$


subject to

$$
\begin{gathered}
\sum_{j:(i, j) \in A} x_{i j} \leq O_{i}, \quad \forall i \in N, \\
\sum_{j:(j, i) \in A} x_{j i} \leq I_{i}, \quad \forall i \in N, \\
x_{i j} \geq 0, \quad \forall(i, j) \in A,
\end{gathered}
$$

which is an inequality constrained transportation problem, and can be solved by distributed auction algorithm [6]. Thus, we can also design a distributed algorithm for sBurst NCC LP, as summarized in Table 8

Table 8. Distributed algorithm on node $j$ for sBurst NCC LP

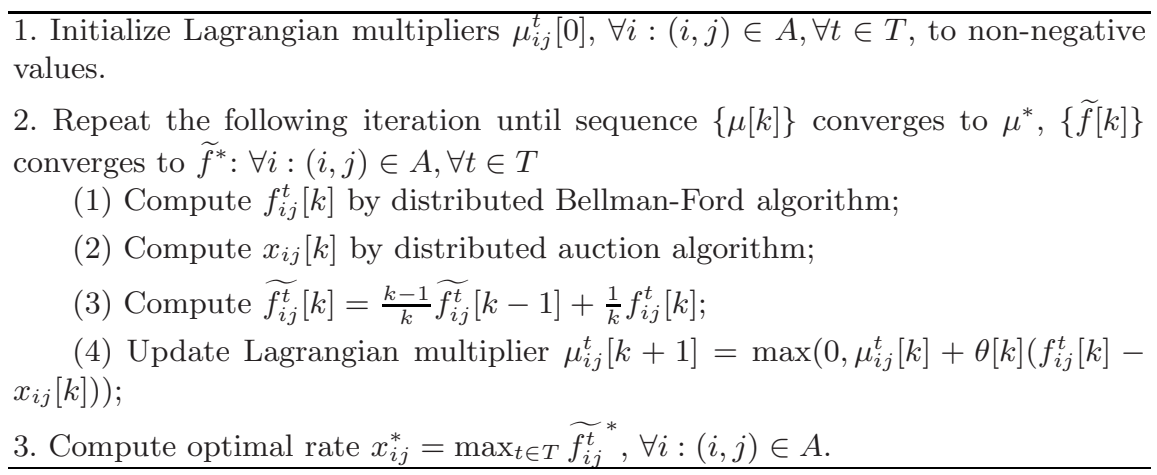

\section{Algorithm Execution in Dynamic Overlays}

In an overlay session characterized by dynamics, the proposed distributed algorithms are also invoked in a dynamic manner. When a node joins a session, it is bootstrapped with a set of upstream nodes. It then starts downloading with the available upload capacities acquired from them. Meanwhile, it requests the source to recompute the optimal rate allocation. When a node departs from a session or fails, an affected downstream node attempts to acquire additional bandwidths from its remaining upstream nodes. Meanwhile, it requests the source to recompute the optimal rate allocation.

At the source, when it receives more than a certain number of requests for recomputation, it broadcasts such a request to all the nodes, which activate a new round of execution of the distributed algorithm, while continuing to download at the original rates. Note that in such a dynamic environment, the execution of a distributed algorithm always starts from the previous optimal values (rather than from the very beginning when all values are initialized to any non-negative values, such as zeros), thus expediting its convergence. After the distributed algorithm converges, all the nodes adjust their download rates to the new optimal values. 


\section{Performance Evaluation}

We next conduct an empirical study of the distributed optimization algorithms. All simulations are conducted over random network topologies generated with BRITE [7] topology generator based on power-law degree distributions. The average number of neighbors per node in the topologies is six. For link-constrained problems, link capacities are generated with heavy-tailed distributions between $100 \mathrm{Kbps}$ and $4 \mathrm{Mbps}$; for node-constrained problems, each node has $1.5-4.5$ Mbps of download capacity and 0.6 - 0.9 Mbps of upload capacity. For sBurst problems, streaming of a $300 \mathrm{Kbps}$ bitstream is simulated and cost coefficients are random numbers chosen from $(0,3)$.

\subsection{Convergence in Static Networks}

To investigate the scalability of our optimal rate allocation algorithms, we first evaluate their convergence speed in static networks of different sizes. For eBurst $L C C$ LP, we have shown in Table 2 a purely combinatorial algorithm, which can derive the solution efficiently. Here, we are more concerned with the efficiency of the iterative subgradient algorithms to solve the other three problems, given in Table 4, 6, 8, respectively.

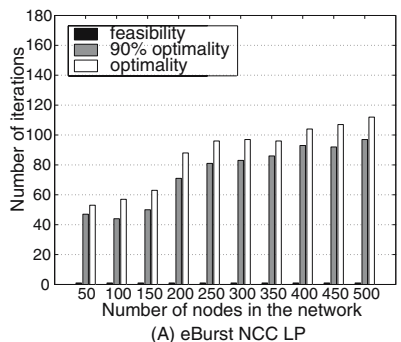

(A) eBurst NCC LP

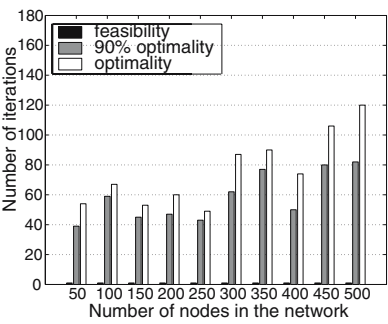

(B) sBurst LCC LP

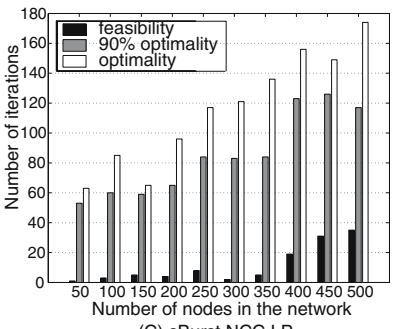

(C) sBurst NCC LP

Fig. 1. Convergence speed in static networks for distributed algorithms in Table 46 8

Fig. 1 shows that for all three problems, with the increase of network sizes, the numbers of iterations their algorithms take to achieve optimality only increase slowly, thus not affecting algorithm scalability. In all cases, the algorithms converge to feasibility within only a few rounds. Even the convergence to $90 \%$ optimality is much faster, within $20 \%$ fewer rounds than those required for convergence to optimality. Therefore, in realistic networks, we can obtain a feasible solution to a certain degree of optimality in a much shorter time, when it is not necessary to achieve absolute optimality.

\subsection{Convergence in Dynamic Networks}

We next investigate the algorithm convergence in practical dynamic environments. Due to space limit, we only show the results obtained by the eBurst NCC algorithm in Table 4, while other algorithms produce similar results. 


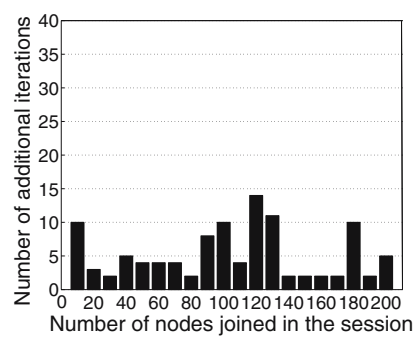

(A) Node joining

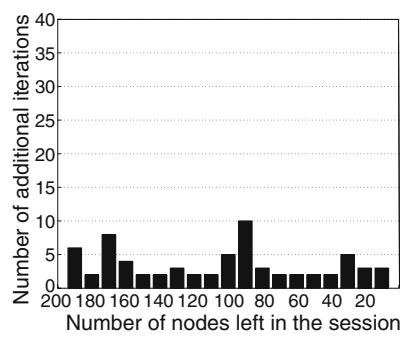

(B) Node leaving

Fig. 2. Convergence speed in a dynamic network for eBurst $N C C$ algorithm in Table 4

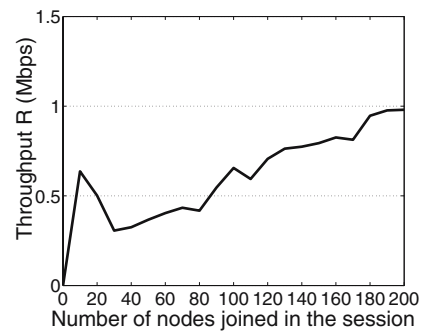

(A) Node joining

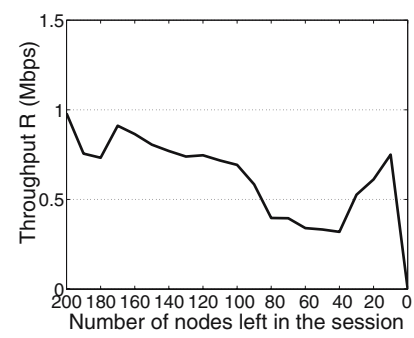

(B) Node leaving

Fig. 3. Throughput achieved in a dynamic network with eBurst $N C C$ algorithm in Table 4

In this experiment, 200 nodes sequentially join an elastic content distribution session, and then start to depart when their downloads are completed. The distributed algorithm is invoked every 10 node joins or departures. As discussed in Sec. 4, the algorithm always runs from the previously converged optimal rates when it is invoked.

We show the number of additional iterations required to converge to new optimal values from the previous ones in node joining and departure phases in Fig. 2(A) and (B), respectively. We find that, compared to running from the very beginning in the cases of static networks of the same sizes, our dynamic execution of the algorithm converges much faster. Independent of the current network sizes, it always takes less than 15 iterations to converge, in both joining and departure cases. While dynamic networks are more akin to realistic scenarios, this suggests our optimal rate allocation algorithms can deliver good performance and provide excellent scalability in practice.

In addition, we illustrate maximum throughput of the dynamic session, $R$, in Fig. 3. In Fig. 3 (A), at the beginning of the node joining phase, the throughput drops because of the competition of more nodes for the available upload capacities in the network. Later, when more nodes have joined, more upload capacities are provided to the session, and thus the throughput gradually increases. During the node departure phase in Fig. 3 (B), due to similar reasons, the throughput first shows a decreasing trend, and then rises when only a few nodes are left. 


\section{Related Work}

On the topic of overlay content distribution, mesh-based approaches have become typical in most recent proposals 8910. Disseminating large-scale content on mesh topologies, their parallel transfers make them possible to deliver at fundamentally higher bandwidth and reliability, without the cost of constructing multicast trees.

With respect to rate allocation in mesh overlay topologies, most existing work either relies on TCP, or employs various heuristics without formulating the problem theoretically. Compared to our optimal rate allocation, their rate allocation falls short of achieving global optimality. There is no way to guarantee that maximum throughput is achieved at all nodes, or a required streaming rate is provided to all at the lowest cost.

There are a few exceptions that formulate the problem into optimization models and propose distributed solutions 111112. Our work is original in that we systematically model all the typical content distribution scenarios, and design efficient algorithms to solve the formulated optimization problem combinatorially or numerically, in a fully distributed manner. In addition, we discuss execution of the algorithms in practical dynamic environments. This has not been addressed in previous optimization-based approaches, most of which are largely theoretical in nature.

\section{Conclusion}

The problem of interest in this paper is to design efficient distributed algorithms for optimal rate allocation under all typical scenarios of overlay content distribution. For this purpose, we formulate rate allocation problems into linear programs, which optimize bandwidth utilization towards a variety of objectives, and develop fully decentralized algorithms to efficiently compute the optimal link rates. We believe such an optimal rate allocation algorithm is critical to any schemes of overlay content distribution. As ongoing work, we are investigating the combination of optimal rate allocation with efficient distribution schemes, and its application in realistic networks.

\section{References}

1. Li, Z., Li, B.: Efficient and Distributed Computation of Maximum Multicast Rates. In: Proc. of IEEE INFOCOM 2005. (March 2005)

2. Ahuja, R.K., Magnanti, T.L., Orlin, J.B.: Network Flows: Theory, Algorithms, and Applications. Prentice Hall (1993)

3. Wu, C., Li, B.: Optimal Rate Allocation in Overlay Content Distribution. Technical report, http://iqua.ece.toronto.edu/papers/ratealloc.pdf (Oct 2006)

4. Bertsekas, D.P., Gallager, R.: Data Networks, 2nd Ed. Prentice Hall (1992)

5. Sherali, H.D., Choi, G.: Recovery of Primal Solutions when Using Subgradient Optimization Methods to Solve Lagrangian Duals of Linear Programs. Operations Research Letter 19 (1996) 105-113 
6. Bertsekas, D.P., Castanon, D.A.: The Auction Algorithm for the Transportation Problem. Annals of Operations Research 20 (1989) 67-96

7. Medina, A., Lakhina, A., Matta, I., Byers, J.: BRITE: Boston University Representative Internet Topology Generator. Technical report, http://www.cs.bu.edu/brite (2000)

8. Kostic, D., Rodriguez, A., Albrecht, J., Vahdat, A.: Bullet: High Bandwidth Data Dissemination Using an Overlay Mesh. In: Proc. of the 19th ACM Symposium on Operating Systems Principles (SOSP) 2003. (October 2003)

9. Sherwood, R., Braud, R., Bhattacharjee, B.: Slurpie: A Cooperative Bulk Data Transfer Protocol. In: Proc. of IEEE INFOCOM 2004. (March 2004)

10. Zhang, X., Liu, J., Li, B., Yum, T.P.: CoolStreaming/DONet: A Data-Driven Overlay Network for Live Media Streaming. In: Proc. of IEEE INFOCOM 2005. (March 2005)

11. Lun, D.S., Ratnakar, N., Koetter, R., Medard, M., Ahmed, E., Lee, H.: Achieving Minimum-Cost Multicast: A Decentralized Approach Based on Network Coding. In: Proc. of IEEE INFOCOM 2005. (March 2005)

12. Adler, M., Kumar, R., Ross, K.W., Rubenstein, D., Suel, T., Yao, D.D.: Optimal Peer Selection for P2P Downloading and Streaming. In: Proc. of IEEE INFOCOM 2005. (March 2005) 